\title{
ANÁLISE DA VARIABILIDADE DA FREQUÊNCIA CARDÍACA EM INDIVÍDUOS HIPERTENSOS SUBMETIDOS À HIDROTERAPIA
}

\author{
Renata França, Nátali Arce \\ Fisioterapeutas graduadas pela UNOESTE, Presidente Prudente, SP.
}

\section{RESUMO}

A hipertensão arterial (HA) é a pressão sistólica maior ou igual a 140 mmHg e diastólica maior ou igual a 90 $\mathrm{mmHg}$, uma condição mórbida presente em grande parte da população. Já a freqüência cardíaca (FC) é modulada por ramos simpático e parassimpático do sistema nervoso autônomo (SNA). As alterações autonômicas podem ser dadas pela análise da variabilidade da freqüência cardíaca (VFC), que determina a preservação ou comprometimento do SNA cardíaco e tem extrema associação com o risco de mortalidade. O objetivo da pesquisa foi observar a resposta da FC de modo a favorecer a interpretação clínica, prescrição de exercícios e prevenção de patologias cardiovasculares. Participaram da pesquisa 15 indivíduos hipertensos, sedentários, faixa etária entre 50 a 75 anos, avaliados por anamnese à quanto medicação e teste de esforço de caminhada para análise do grau de estratificação de risco e melhor programação de tratamento. O tratamento fisioterápico foi realizado na piscina aquecida da Clínica de Fisioterapia da Universidade do Oeste Paulista - UNOESTE de Presidente Prudente. A execução da atividade física foi realizada com intensidade de 60 a 75\% da freqüência cardíaca submáxima (195 - idade) utilizando a fórmula Karvonen. Foi utilizado o teste $T$ para dados pareados. Resultados mostraram-se significativos nos intervalos RR $(p<0,005)$ e não houve respostas significativas nos intervalos RMSSD e PNN50 ( $p>0,005)$. A pesquisa revelou que não houve alteração na VFC após o período de treinamento, supondo-se que o período de férias e ausência de alguns pacientes no tratamento pode ter interferido nas respostas.

Palavras-chave: Variabilidade da freqüência cardíaca, hipertensão, hidroterapia.

\section{ANALYSIS OF HEART RATE VARIABILITY IN HYPERTENSIVE INDIVIDUALS WITHIN THE HYDROTHERAPY}

\begin{abstract}
Arterial hypertension $(\mathrm{AH})$ is the systolic pressure greater than or equal to $140 \mathrm{mmHg}$ and diastolic blood pressure greater than or equal to $90 \mathrm{mmHg}$, a morbid condition present in a large population. Since the heart rate $(\mathrm{HR})$ is modulated by sympathetic and parasympathetic branches of the autonomic nervous system (ANS). Autonomic changes may be given by the analysis of heart rate variability (HRV), which determines the preservation or impairment of cardiac SNA, witch is extremely associated with the risk of mortality. The purpose of this research was to observe the response of HR in order to facilitate clinical interpretation, exercise prescription and prevention of cardiovascular diseases. The participants were 15 hypertensive, sedentary, age 50 to 75 years, assessed medical history, medication and stress test analysis of walking to the degree of risk stratification and better planning of treatment. The physiotherapy treatment was performed in the heated pool of physical therapy clinic at the University of Oeste Paulista - UNOESTE of Presidente Prudente. The implementation of physical activity was performed with 60 to $75 \%$ of submaximal heart (195 age) using the Karvonen formula. It was used T test for paired data. Results proved significant in RR intervals $(p<0.005)$ and no significant responses in the intervals and RMSSD and PNN50 $(p>0.005)$. The survey revealed that there was no change in HRV after the training period, assuming that the holiday period and the absence of treatment in some patients may have influenced the responses.
\end{abstract}

Keywords: Heart rate variability, hypertension, hydrotherapy. 


\section{INTRODUÇÃO}

A definição da hipertensão arterial (HA) é dada como pressão sistólica maior ou igual a 140 $\mathrm{mmHg}$ e diastólica maior ou igual a $90 \mathrm{mmHg}$, considerada uma condição mórbida presente na população brasileira, responsável por $40 \%$ dos óbitos no país. Estima-se que entre vinte a trinta milhões de pessoas sejam hipertensas entre crianças e adultos no Brasil (FARINATTI, 2002).

A HA é causada por aumento da atividade simpática e diminuição da atividade vagal. Portanto, o reajuste do balaço simpatovagal no coração com aumento da modulação vagal é uma das mudanças adaptativas na regulação neural produzida pelo exercício físico que pode ser observado pelo aumento da variabilidade da freqüência cardíaca (VFC) nos hipertensos (NOVAIS et al., 2004).

O estudo da VFC é um método nãoinvasivo que analisa a modulação do sistema nervoso autônomo (SNA) em certas condições fisiológicas, tais como em situações de vigília e sono, diferentes posições do corpo, treinamento físico e também em condições patológicas (CATAl et al., 2002).

A estimativa indireta de alterações autonômicas no sistema cardiovascular pode ser dada pela análise da VFC e pela pressão arterial (PA) (SILVA; JANUÁRIO, 2005).

O conhecimento da resposta da freqüência cardíaca (FC) nas diversas situações de exercício torna-se essencial para a correta prescrição e posterior controle das cargas de treinamento aeróbio, assim como a identificação dos métodos e modelos de treinamento de força que resultam em sobrecarga cardíaca (ALMEIDA; ARAÚJO, 2003).

A FC é modulada por uma ação conjunta, embora independente, dos ramos simpático e parassimpático do SNA. Ao inibir parcial ou completamente a atividade vagal cardíaca, ocorre aceleração da FC (CONSTANZO, 2004).

As evidências indicam que a $F C$ de repouso tende a refletir condição de saúde, pois indivíduo com FC de repouso baixa tem um prognóstico mais favorável em termos de risco de mortalidade. Entretanto, existe uma baixa associação com a condição aeróbia. Supõe-se que a razão para valores menores em repouso seja em função de maior atividade vagal nesse período. Esta é uma característica dos atletas de endurance (GREENLAND et al.,1999).

Em indivíduos com função cardiovascular comprometida, como por exemplo, os hipertensos, a FC de repouso diminuída pode representar maior perfusão coronariana. A irrigação do músculo cardíaco ocorre primariamente durante a diástole, pois a localização epicárdica das artérias coronárias faz com que sejam ocluídas durante a sístole. Sendo assim, um ritmo cardíaco mais lento gera um período de tempo diastólico mais longo e favorece a perfusão coronariana (BRAITH; EDWARDS, 2003).

Como conseqüência das adaptações constantes promovidas pelo SNA para manter o equilíbrio do sistema cardiovascular, a freqüência cardíaca varia batimento a batimento (MENEZES et al., 2004). Estas alterações podem ser avaliadas por meio das variações nos intervalos $\mathrm{RR}$, constituindo assim a variabilidade da freqüência cardíaca (TASK FORCE, 1996).

A VFC determina a preservação ou comprometimento do SNA cardíaco e tem extrema associação com o risco de mortalidade (ROBINSON et al., 2003). Considera-se a atividade vagal cardíaca como um potente e independente indicador prognóstico em indivíduos com ou sem doença cardiovascular (BUCH et al., 2002). Portanto, a diminuição da VFC pode ser um indicador prognóstico de algumas doenças 
cardíacas e sistêmicas (GRUPI et al., 1994) e uma alta VFC indica um bom funcionamento dos mecanismos de controle autonômico (PUMPRLA et al., 2002).

Alguns estudos correlacionam a diminuição da VFC com a idade à perda de condicionamento físico e ao envelhecimento e, que esta situação poderia ser revertida com a melhora da condição física aeróbia, outros sugerem que a idade isoladamente seria 0 principal fator de diminuição da modulação autonômica (ARAÚJO; ALMEIDA, 2003).

A VFC é mais evidente no repouso do que no exercício. É um marcador da atividade autonômica, ou seja, quanto maior a oscilação da FC em repouso, maior a participação vagal (ALMEIDA; ARAÚJO, 2003). A FC em repouso recebe maior influência colinérgica, desacelerando o ritmo cardíaco (VOGUEL et al., 2004).

Se considerarmos que, com o aumento da intensidade do exercício ocorre aumento da estimulação simpática e concomitante diminuição da estimulação parassimpática, seria de esperar que no pico do esforço a VFC fosse nula ou quase nula. Utilizando protocolo de rampa em cicloergômetro, a VFC no domínio do tempo (DT) é mantida ao longo de todo o exercício máximo, no pico de esforço. Isto pode ser explicado por mecanismos hemodinâmicos, especial o lusitropismo (propriedade de relaxamento do miocárdio). Essa maior capacidade de relaxamento tende a aumentar a diferença de pressão entre as veias e as câmaras cardíacas, favorecendo o retorno venoso e aumentando a pré-carga (ALMEIDA et al., 2005).

Em consequência, há aumento na contratilidade miocárdica que, associada aos mecanismos periféricos auto-reguladores da póscarga, resulta num conjunto de fatores responsável por alterações sucessivas no volume sistólico (SAGI et al., 2000). Para se manter o mesmo aporte sanguíneo via débito cardíaco, a FC também se mantém variável. Portanto, os mecanismos de controle da VFC no exercício máximo parecem não ser exclusivamente autonômicos (PERINI; VEICSTEINAS, 2003; CASTIES et al., 2006).

Apesar de observarmos na prática maior queda na velocidade da FC pós-exercício, não existem evidências que este efeito possa aumentar a cardioproteção vagal (YAWN et al., 2003). O treinamento físico do tipo aeróbico promove um aumento na VFC em indivíduos idosos, porém, os efeitos do treinamento físico do tipo anaeróbico, como o treinamento isométrico e o de força, sobre a VFC em indivíduos de diferentes faixas etárias apresentam resultados ainda inconclusivos (LOPES et al., 2007).

A prática do exercício físico regular auxilia no controle da PA em curto e longo prazo, sendo indicado como intervenção adjunta no manejo da hipertensão arterial (CHOBANIAN et al., 2003). Pode promover a queda sustentada dos níveis pressóricos no pós-esforço, um efeito denominado hipotensão pós-exercício, o qual está em relação ao exercício aeróbico (KENNEY; SEALS, 1993).

Exercícios aeróbios são utilizados como meio terapêutico visando à redução dos níveis pressóricos em indivíduos hipertensos (LIMA et al., 1998). Associado a isso, indivíduos hipertensos, fisicamente ativos, apresentam taxas de mortalidade menores que as dos sedentários (PINTO et al., 2003).

Em relação à hidroterapia é um método terapêutico que utiliza os princípios físicos da água em conjunto com a cinesioterapia. Trabalha grandes grupos musculares e várias articulações ao mesmo tempo com o trabalho aeróbico (CANDEROLO; SILVA, 2003).

A turbulência proporciona relaxamento e propriocepção ao paciente e a viscosidade 
proporciona reeducação muscular e resistência (HANSON; NORM, 1998).

Em relação à pressão hidrostática, a lei de Pascal estabelece que a pressão de fluido seja exercida igualmente sobre todas as áreas do corpo imerso em uma dada profundidade. Essa diferença de pressão na posição vertical precipita um movimento dos fluidos corporais da região distal para a proximal, uma reação diurética ocorre na imersão em decorrência da expansão de volume central que supre o hormônio antidiurético e a combinação de pressão hidrostática e exercícios apropriados do membro aumenta a circulação. Todos esses fatores contribuem para a reabsorção de edemas, além de oferecer resistência na musculatura respiratória (CAMPION, 1999).

A hidroterapia também proporciona modificações fisiológicas como aumento da freqüência respiratória e cardíaca, aumento da circulação periférica, o que leva ao maior suprimento sanguíneo para o músculo, aumentando consequentemente ao metabolismo muscular. Aumenta o retorno sanguíneo ao coração e diminui a pressão arterial, o edema pela pressão hidrostática e a sensibilidade dos terminais nervosos, proporcionando relaxamento muscular geral (THOMSON et al., 1994). Porém, ocorre diminuição gradativa da FC conforme aumenta a profundidade de imersão, durante a imersão em pé no meio aquático (GRAEF; KRUEL, 2006).

Dessa maneira, o presente estudo teve como objetivo analisar a VFC em indivíduos hipertensos no DT, um método de análise de VFC simples, que mostra que a FC é determinada em qualquer ponto no tempo ou nos intervalos RR, de um eletrocardiograma (ECG), que estabelece o complexo QRS. Revelando o comportamento do SNA de modo a favorecer a interpretação clínica, prescrição de exercícios e prevenção diante de fatores de riscos cardiovasculares ao esforço.

\section{MÉTODOS}

Foram estudados 15 pacientes de ambos os sexos, idade entre 50 e 75 anos, com diagnóstico de $H A$, sem uso de qualquer medicação e sedentários. O projeto foi aprovado pelo Comitê de Ética em Pesquisa, os pacientes selecionados foram informados do estudo e assinaram Termo de Consentimento Livre e Esclarecido. Posteriormente foi realizada anamnese dos pacientes e teste de esforço de caminhada de 6 minutos, para análise do grau de estratificação de risco e programação do tratamento.

O tratamento fisioterapêutico foi realizado na piscina aquecida da Clínica de Fisioterapia da Universidade do Oeste Paulista - UNOESTE de Presidente Prudente em sessões de 60 minutos, dois dias por semana, durante 14 meses, totalizando 60 sessões fisioterapêuticas. A execução da atividade física dinâmica foi com intensidade de 60 a 75\% da FC submáxima (195 idade) utilizando a fórmula Karvonen de treinamento (FCT $=\mathrm{FCR}+60 \%$ a $75 \%$ (FC submáx. - FCR) (REGENGA, 2000).

O exercício físico dinâmico foi iniciado com aquecimento de 5 minutos de autoalongamento de membros superiores (MMSS) e de membros inferiores (MMII) com duração de 30 segundos cada alongamento e repouso de 10 segundos na água, seguindo com 5 minutos de caminhada leve com exercícios ativos de MMSS e tronco, associados a respirações com freno labial e 30 minutos de caminhada moderada + diagonais de Kabatt + flexão de ombro com bastão, bola, halteres e espaguetes próprios para hidroterapia. No desaquecimento seguiu-se o mesmo programa do aquecimento (KISNER; COLBY, 2005). 
Foi colocada, no tórax dos pacientes, a cinta de captação e, no seu pulso, o receptor de FC Polar S810, equipamento validado para captação da FC de batimento a batimento e utilização dos seus dados para análise de VFC. Este equipamento consiste em 2 eletrodos montados em um transmissor eletrônico selado posicionado no tórax do indivíduo, ao nível do terço distal do esterno, utilizando uma cinta elástica que capta os impulsos elétricos cardíacos, transmitindo informações de um campo eletromagnético para o monitor no pulso do participante. A FC foi analisada durante 30 minutos em repouso no primeiro tratamento $\left(1^{\text {a }}\right.$ sessão), logo após a avaliação e após o exercício no ultimo tratamento ( $60^{\mathrm{a}}$ sessão). Durante a pesquisa a FC foi verificada de 5 em 5 minutos durante todo tratamento pelo Polar FS1.

Os dados foram analisados pelo DT para análise da VFC os índices RMSSD e pNN50 (MARÃES et al., 2004). Este índice é um marcador sensível e facilmente interpretável da atividade do SNA parassimpático, definido como a percentagem das diferenças sucessivas do intervalo R-R cujo valor absoluto excede $50 \mathrm{~ms}$ (BURR et al., 2003).

Foi utilizada a Estatística Descritiva e os resultados foram analisados usando teste $t$ de Student pareado, para comparação do mesmo grupo de pacientes, antes e após o tratamento. Os dados foram apresentados como média \pm desvio padrão, o nível de significância foi de 5\% e intervalo de confiança de $95 \%$.

\section{RESULTADOS}

O trabalho foi realizado com 15 voluntários, sendo 7 homens e 3 mulheres (66\%), mais 5 pacientes (34\%) que desistiram do tratamento.

A Tabela 1 mostra os dados, na fase prétratamento e pós-tratamento, dos índices SDNN, RMSSD e Média RR. Na fase pós-tratamento houve diminuição dos valores, porém não significativa.

Tabela 1. Valores da média, desvio padrão nas fases pré e pós-tratamento.

\begin{tabular}{lccc}
\hline Índice & Pré-tratamento & Pós-tratamento & $\mathbf{p}$ \\
\hline SDNN & $0,023 \pm 0,008$ & $0,021 \pm 0,008$ & 0,424 \\
RMSSD & $16,35 \pm 6,89$ & $15,07 \pm 7,35$ & 0,399 \\
Média & $0,81 \pm 0,11$ & $0,79 \pm 0,11$ & 0,023 \\
RR & & & \\
\hline
\end{tabular}

A Tabela 2 refere-se aos valores mínimos e máximos nas respectivas fases e índices.

Tabela 2. Diferença dos valores mínimos e valores máximos nas fases pré e pós-tratamento.

\begin{tabular}{lcc}
\hline Índice & Pré-tratamento & Pós-tratamento \\
\hline SDNN & $0,01-0,04$ & $0,00-0,04$ \\
RMSSD & $7,8-31,0$ & $2,3-30,9$ \\
Média RR & $0,60-1,02$ & $0,59-1,00$ \\
\hline
\end{tabular}

\section{DISCUSSÃO}

Segundo estudos, a diminuição da VFC pode indicar prognóstico de algumas doenças cardíacas e sistêmicas (GRUPI et al., 1994) e alta VFC indica um bom funcionamento do controle autonômico (PUMPRLA et al., 2002). Entretanto alguns estudos correlacionam a diminuição da VFC com uma redução condicionamento físico ao envelhecimento e assim, ser revertida com a melhora da condição física aeróbia (ARAÚJO; ALMEIDA, 2003).

Para Vanderlei et al. (2009) as facilidades para aquisição de dados fazem da VFC uma opção interessante para interpretações do funcionamento do SNA e uma ferramenta clínica promissora para avaliar e identificar comprometimentos na saúde. 
Contudo neste estudo não ocorreram resultados estatisticamente significativos dos índices temporais (RMSSD e pNN50) ou espectrais (HF, LF e a relação LF/HF) de VFC da VFC, sendo nos índices RMSSD e SDNN $(P>0,05)$. Obteve-se resultados significativos apenas na média RR $(P<0,05)$, após 0 cumprimento do tratamento. Boutcher et al. (1997) sugerem que o exercício aeróbio modula beneficamente a VFC na população em envelhecimento, tal redução pode ser devido ao aumento da atividade parassimpática, que conduz para um aumento da duração do intervalo RR.

Paschoa et al. (2006) mostraram redução da variabilidade de $R-R$, ao contrário ao presente estudo, porém com componente estável. Sendo possível uma menor proteção da modulação vagal cardíaca, o que alerta quanto ao risco de graves eventos cardiovasculares quando a realização de exercícios com características semelhantes.

Já na presente pesquisa observou-se um aumento da VFC apenas no intervalo RR, o que indica uma boa modulação autonômica cardíaca.

Para Lopes et al. (2007), após o cumprimento do programa de treinamento físico aeróbio, durante aproximadamente três anos, houve um aumento de todas as variáveis utilizadas para análise da VFC, indicando uma mudança na modulação autonômica, embora essas modificações não tenham sido estatisticamente significativas.

Assim como, na presente pesquisa, Boutcher e Stein (1995), não relataram mudanças nas medidas do domínio da freqüência da VFC em um grupo de homens sedentários de meia idade velhos, com 8 semanas de treinamento aeróbio, apesar de significante diminuição na FC de repouso.

Marães et al. (2003) e Araújo e Almeida (2003) observaram um aumento da VFC em repouso, após o exercício de alta intensidade, que modula a atividade autonômica do sistema nervoso sobre o coração.

Pichon et al. (2004), demonstraram que o exercício pode equilibrar a atividade autonômica, devido à redução da FC de repouso e aumentando a VFC. E assim, diminuindo risco de arritmias e morte súbita em seres humanos e animais. A pesquisa atual relatou semelhança no equilíbrio da atividade autonômica.

Paschoal et al. (2003), observou que, com exercício ocorre o aumento da atividade simpática e diminuição da atividade parassimpática, no índice de RMSSD, demonstrando a ação parassimpática sobre o coração com o exercício.

Segundo Aubert et al. (2003), o treino aeróbico de intensidade moderada e curta duração em uma população de meia idade são insuficientes para alterar a VFC. Ainda segundo Loimaala (2000), o programa de treino deve durar um período de pelo menos um ano, para que se obtenha efeito sobre a VFC.

Esta pesquisa revelou que houve mudanças significativas da VFC na média RR, após o período de treinamento. Mas o período de férias e ausência de alguns pacientes no tratamento pode ter interferido nas respostas da VFC.

Desse modo, sugere-se futuras investigações sobre a VFC de modo favorecer a interpretação clínica e prescrição de exercício, prevenção diante de fatores de risco ou mesmo possibilitando a descoberta de diagnósticos cardiovasculares de esforço.

\section{REFERÊNCIAS}

Almeida MB, Araújo CGS. Efeitos do treinamento aeróbio sobre a freqüência cardíaca. Revista Brasileira de Medicina do Esporte 2003; 9:104-12.

Almeida MB, Ricardo DR, Araújo CGS. Variabilidade da freqüência cardíaca em uma tese de exercício verdadeiramente máximo. Revista SOCERJ 2005; 18(6):534-41. 
Araújo CGS, Almeida MB. Efeitos do treinamento físico sobre a freqüência cardíaca. Revista Brasileira de Medicina do Esporte 2003; 9(2):10412.

Aubert AE, Seps B, Beckers F. Heart rate variability in athletes. Sports Medicine 2003; 12(33):889-919.

DOI: http://dx.doi.org/10.2165/00007256-200333120$\underline{00003}$

Boutcher SH, Cotton Y, Nurhayati Y, Craig GA, Mclaren PF. Autonomic nervous function at rest in aerobically trained and untrained older men. Clinical Physiology 1997; 17:339-46. DOI: http://dx.doi.org/10.1046/i.13652281.1997.03838.x

Boutcher SH, Stein P. Association between heart rate variability and training response in sedentary middle-aged men. European Journal of Applied Physiology and Occupational Physiotherapy 1995; 70:75-80.

DOI: http://dx.doi.org/10.1007/BF00601812

Braith RW, Edwards DG. Neurhormonal abnormalities in heart failure:impact of exercise training. Congestive Heart Failure 2003; 9:70-6. DOI: $\underline{5299.2003 .00277 . x}$

Buch NA, Coote JH, Townend JN. Mortality, cardiac vagal control and physical training-what's the link? Experimental Physiology 2002; 87:42335.

Burr RL, Motzer SA, Chen W, Cowan MJ, Heitkemper MM. A nonlinear transformation of pNN50 with improved statistical properties. Journal of Electrocardiology 2003; 36:41-52. DOI: http://dx.doi.org/10.1054/jelc.2003.50009

Campion M. Hidroterapia: princípios e prática. São Paulo: Manole; 1999.

Canderolo JM, Silva RR. Proposta de protocolo hidroterapêutico para fraturas de fêmur na terceira idade. [documento eletrônico] 2003. Acesso em 23 out 2008]. Disponível em: www.poolterapia.com.br

Casties JF, Mottet D, Lê Gallais D. Non-linezr analyses of heart rate variability during heavy exercise and recovery in cyclists. International Journal of Sports Medicine 2006; 27:780-5. DOI: http://dx.doi.org/10.1055/s-2005-872968

Catai AM, Chacon-Mikahil MPT, Martinelli FS, Forti VAM, Silva E, Golfetti R, et al. Effects of aerobic exercise training on heart rate variability during wakefulness and sleep and cardiorespiratory responses of young and middle- age healthy men. Brazilian Journal of Medicine and Biological Research 2002; 35:741-752. DOI: http://dx.doi.org/10.1590/S0100$\underline{879 \times 2002000600016}$

Chobanian AV, Bakris GL, Black HR, Cushman WC, Green LA, Izzo JLJ. The Seventh Report of the Joint National Committee on Prevention, Detection, Evaluation, and Treatment of High Blood Pressure: the JNC 7 report. JAMA 2003; 289(19):2560-72.

http://dx.doi.org/10.1001/jama.289.19.2560

Constanzo L. Fisiologia. 2ed. Rio de Janeiro: Elsevier; 2004.

Farinatti PTV. Aspectos da prescrição do exercício para hipertensos. Revista Brasileira de Medicina do Esporte 2002; 1(1):123-42.

Graef FI, Kruel LFM. Freqüência cardíaca e percepção subjetiva do esforço no meio aquático: diferenças em relação ao meio terrestre e aplicações na prescrição do exercício - uma revisão. Revista Brasileira de Medicina do Esporte 2006; 12(4):221-8. DOI: http://dx.doi.org/10.1590/S1517$\underline{86922006000400011}$

Greenland P, Daviglus ML, Dyer AR, Liu K, Hang $\mathrm{CF}$, Goldberger JJ. Resting heart rate is a risk factor for cardiovascular and noncardiovascular mortality: the Chicago Heart Association Detection Project in Industry. American Journal of Epidemiology 1999; 149:853-62. DOI: http://dx.doi.org/10.1093/oxfordjournals.aje.a0099 $\underline{01}$

Grupi C, Moffa PJ, Barbosa AS, Sanches PCR, Barragam FEG, Pileggi F. Variabilidade da freqüência cardíaca: significado e aplicação clínica. Revista da Associação Médica Brasileira 1994; 40(2):129-36.

Hanson B, Norm A. Exercícios Aquáticos Terapêuticos. São Paulo: Manole; 1998.

Kenney MJ, Seals DR. Postexercise hypotension: key features, mechanisms, and clinical significance. Hypertension 1993; 22(5):653-64. DOI: http://dx.doi.org/10.1161/01.HYP.22.5.653

Kisner C, Colby LA. Exercícios Terapêuticos: fundamentos e técnicas. São Paulo: Manole; 2005.

Lima EG, Herkenhoff F, Corral EV. Monitorização ambulatorial da pressão arterial em indivíduos com resposta exagerada dos níveis pressóricos em esforço. Influência do condicionamento físico. Arquivos Brasileiros de Cardiologia 1998; 70(4):243-9.
DOI: 
http://dx.doi.org/10.1590/S0066782X1998000400002

Loimaala A, Huikuri H, Oja P, Pasanen M, Vouri I. Controled 5-mo aerobic training improves heart rate but not heart rate variability or baroreflex sensitivity. Journal of Applied Physiology 2000; 89:1825-29.

Lopes FL, Pereira FM, Reboredo MM, Castro TM, Vianna JM, Novo JJM, Silva LP. Redução da Variabilidade da freqüência cardíaca em indivíduos de meia-idade e o efeito do treinamento de força. Revista Brasileira de Fisioterapia 2007; 11(2):113-9. DOI: http://dx.doi.org/10.1590/S1413$\underline{35552007000200005}$

Marães VRSF, Santos MDB, Catai AM, Moraes FR, Oliveira L, Gallo Jr L, Silva E. Modulação do sistema nervoso autonômico na resposta da freqüência cardíaca em repouso e à manobra de Valsalva com o incremento da idade. Revista Brasileira de Fisioterapia 2004; 8(2):97-103.

Marães VR, Teixeira LC, Catai AM, Milan LA, Rojas FA, De Oliveira L. Determinação e validação do limiar de anaerobiose a partir de métodos de análise da freqüência cardíaca e de sua variabilidade. Revista da SOCESP 2003;13 (4 Suppl A):S1-16.

Menezes JRAS, Moreira HG, Daher MT. Análise da variabilidade da freqüência cardíaca em pacientes hipertensos, antes e depois do tratamento com inibidores da enzima conversora da angiotensina II. Arquivos Brasileiros de Cardiologia, São Paulo 2004; 83(2):165-8. DOI: http://dx.doi.org/10.1590/S0066$\underline{782 \times 2004001400008}$

Novais LD, Sakabe DI, Takahashi ACM, Gongora $\mathrm{H}$, Taciro C, Martins LEB, Oliveira L, Silva E, Gallo JL, Catai AM. Avaliação da variabilidade da freqüência cardíaca em repouso de homens saudáveis sedentários e de hipertensos e coronariopatias em treinamento físico. Revista Brasileira de Fisioterapia 2004; 8(3):207-13.

Paschoa DC, Coutinho JFS, Almeida MB. Análise da VFC no exercício de força. Revista SOCERJ 2006; 19:385-90.

Paschoal MA, Gonçalves NVO, Petrelluzzi KFS, Machado RV. Controle autonômico cardíaco durante a execução de atividade física dinâmica de baixa intensidade. Revista SOCESP 2003; 13(5 Supl A):1-11.

Perini $R$, Veicsteinas A. heart rate variability and autonomic activity at rest and during exercise in various physiological conditions. European
Journal of Applied Physiology 2003; 90:317-25. DOI: http://dx.doi.org/10.1007/s00421-003-0953-9

Pichon AP, Bissochop C, Roulaud M, Denjean A, Papelier Y. Spectral analysis of heart rate variability during exercise in trained subjects. Medicine and Science in Sports \& Exercise 2004; 36(10):1702-8.

DOI: http://dx.doi.org/10.1249/01.MSS.0000142403.93 $\underline{205.35}$

Pinto VLM, Meirelles LR, Farinatti PTV. Influência de programas não formais de exercícios (doméstico e comunitário) sobre a aptidão física, pressão arterial e variáveis bioquímicas em pacientes hipertensos. Revista Brasileira de Medicina do Esporte 2003; 9(5):267-74.

Pumprla J, Howorka K, Groves D, Chester M, Nolan J. Functional assessment of heart rate variability: physiological basis and practical applications. International Journal of Cardiology 2002; 84:1-14. DOI: http://dx.doi.org/10.1016/S0167-5273(02)00057-8

Regenga, MM. Fisioterapia em cardiologia: da unidade de terapia intensiva à reabilitação. São Paulo: Roca; 2000.

Robinson TG, Dawson SL, Eames PJ, Panerai RB, Potter JF. Cardiac baroreceptor sensitivity predicts long-term outcome after acute ischemic stroke. Stroke 2003; 34:705-12. DOI: http://dx.doi.org/10.1161/01.STR.0000058493.948 $\underline{75.9 F}$

Sagi M, Bem-Sira D, Goldhammer E, Soundry M. Left ventricular contractility and function at peak aerobic and anaerobic exercises. Medicine and Science in Sports \& Exercise 2000; 32:1197-201. DOI: $\quad$ http://dx.doi.org/10.1097/00005768200007000-00002

Silva VJD, Januário EN. Heart rate and arterial pressure variability in congestive heart failure. Revista Brasileira de Hipertensão 2005; 12(1):216.

Task Force of the European Society of Cardiology and the North American Society of Pacing and Electrophysiology. Heart rate variability: standards of measurement, physiological interpretation, and clinical use. European Heart Journal 1996; 17:354-81.

DOI: http://dx.doi.org/10.1093/oxfordjournals.eurheartj. $\underline{\mathrm{a} 014868}$

Thomson A, Skinner A, Piercy J. Fisioterapia de Tidy. São Paulo: Editora Santos; 1994.

Vanderlei LCM, Pastre CM, Hoshi RA, Carvalho TD, Godoy MF. Noções básicas de variabilidade da frequência cardíaca e sua aplicabilidade 
clínica. Revista Brasileira de Cirurgia Cardiovascular 2009; 24(2):205-17. DOI: http://dx.doi.org/10.1590/S0102-

$\underline{76382009000200018}$

Voguel CU, Wolpert C, Wehling M. How to measure heart rate? European Journal of Clinical Pharmacology 2004; 60:461-6. DOI: http://dx.doi.org/10.1007/s00228-004-0795-3

Yawn B, Ammar K, Thomas R, Wollan P. TestRetest Reproducibility of Heart Rate Recovery After Treadmill Exercise. Annals of Family Medicine 2003; 1:236-41. DOI:

http://dx.doi.org/10.1370/afm.37 